A $\mathrm{C} \cup$ Rec. Nat. Prod. 15:4 (2021) 301-311

\title{
Volatile Constituents of Cistanche tubulosa and Their Antioxidant
}

\section{and Antimicrobial Potentials}

\author{
Arafa Musa 1,2*, Khaled F. El-Massry $\oplus^{3,4}$, Ahmed H. El-Ghorab ${ }^{3,4}$, Amr \\ Farouk $\oplus^{4}$, Hazim M. Ali $\odot^{3}$, Mohamed A Abdelgawad ${ }^{5,6}$, \\ Ibrahim A. Naguib $\oplus^{7}$ and Ehab M. Mostafa $\odot 1,2$
}

\author{
${ }^{1}$ Department of Pharmacognosy, College of Pharmacy, Jouf University, Sakaka, Aljouf 72341, Saudi \\ Arabia \\ ${ }^{2}$ Department of Pharmacognosy, Faculty of Pharmacy, Al-Azhar University, Cairo, 11371, Egypt \\ ${ }^{3}$ Chemistry Department, College of Science, Jouf university, Sakaka, Aljouf 72341, Saudi Arabia \\ ${ }^{4}$ Flavour and Aroma Department, National Research Center, Dokki, Giza, Egypt \\ ${ }^{5}$ Department of Pharmaceutical Chemistry, College of Pharmacy, Jouf university, Sakaka, Aljouf 72341, \\ Saudi Arabia \\ ${ }^{6}$ Department of Pharmaceutical Organic Chemistry, Faculty of Pharmacy, Beni-Suef university, Beni- \\ Suef 62514, Egypt \\ ${ }^{7}$ Department of Pharmaceutical Chemistry, College of Pharmacy, Taif University, P.O. Box 11099, Taif \\ 21944, Saudi Arabia
}

(Received November 02, 2020; Revised December 13, 2020; Accepted December 19, 2020)

\begin{abstract}
The hydrodistilled volatile constituents of Cistanche tubulosa (commonly known as Desert Ginseng) have been chemically and biologically investigated. Based on the retention times and mass fragmentation of the obtained GC-MS chromatogram, 106 individual components which representing $\approx 99.29 \%$ of the total volatile constituents have been identified. The major compounds (66.57\% of the total composition) were identified as hexanal (15.98\%), trans-sabinyl acetate (12.22\%), allo-aromadendrene (9.30\%), nonanoic acid (6.66\%), 3Zhexeny-2-methyl butanoate $(6.09 \%)$, valeranone $(5.25 \%),(E, E)$ - $\alpha$-Farnesene $(3.18 \%)$, $\alpha$-pinene $(3.06 \%)$, linalool isovalerate $(3.03 \%)$ and $\alpha$-humulene $(1.8 \%)$. Estimation of the antioxidant activity of EO showed promising effect at $80 \mu \mathrm{g} / \mathrm{mL}$ concentration, it exerted $62.40,863.29$ and $62.72 \%$ inhibition compared to TBHQ that showed 78.62 , 77.56 and $79.23 \%$ inhibition using DPPH, ABTS and $\beta$-carotene/Linoleic acid, respectively. The antioxidant activity was pronounced at $80 \mu \mathrm{g} / \mathrm{mL}$ than other concentrations. The volatile constituents showed inhibitory activity against gram positive bacteria ranged from $2.23 \mathrm{mg} / 100 \mathrm{~mL}$ ( for staphylococcus aureus ), and $15.68 \mathrm{mg} / 100 \mathrm{~mL}$ (for Bacillus cereus) compared to ciprofloxacin which showed inhibitory activity 0.185 , and $0.182 \mathrm{mg} / 100 \mathrm{~mL}$, respectively. Moreover, the MIC of volatiles towards gram negative bacteria are ranged from 18.35 (Escherichia coli) to $31.61 \mathrm{mg} / 100 \mathrm{~mL}$ (Klebsiella pneumonia) compared to ciprofloxacin with 0.184 to $0.188 \mathrm{mg} / \mathrm{mL}$ respectively. Additionally, the antifungal activity against candida albicans was rather promising $(4.36 \mathrm{mg} / \mathrm{mL})$.
\end{abstract}

Keywords: Cistanche tubulosa; volatile constituents; antioxidant; antimicrobial; Orobanchaceae. . (C) 2021 ACG Publications. All rights reserved.

\footnotetext{
* Corresponding author: E-Mail: akmusa@ju.edu.sa
} 


\section{Introduction}

Cistanche tubulosa, family Orobanchaceae, is a perennial parasitic plant, growing in arid areas of Asia and Africa, it has been detected in China, India, Japan, Saudi Arabia (Sakaka desert, Aljouf, KSA) [1]. It possesses various common names in Chinese medicine as Desert Hyacinth, Desert ginseng and Rou Cong Rong, the stem is succulent and fleshy with high water content [1-6]. Cistanche is familiar for volatile and non-volatile constituents that may include lignans, phenylethanoid glycosides, oligo- and polysaccharides, alkaloids and iridoids. Due to the great diversity of the phytochemical content and biological activities, Cistanche has acquired a high medicinal value in Chinese folk and traditional medicines. Consequently, it has been used as aphrodisiac in case of impotence and infertility, laxative in senile constipation, and found to have neuroprotective effect especially in case of Alzheimer's, Parkinson's and depression, anti-aging, anti-neoplastic, antiplatelet aggregation, antifungal and antibacterial, hepatoprotective, immunostimulant, antioxidant, renal support, and antitumor in colorectal esophageal carcinoma [1, 7-13]. It is also used in treatment of psychroalgia of the knees and back, improvement of immunity and cognitive activities and as antidepressant [14-17]. It has been found to have hypocholesterolemic effect as reported by Shimoda et al [18]. The literature survey declared the safe use of Cistanche as non-toxic plant for the long run [19]. Although C. tubulosa was reputed by the high medicinal values particularly in Chinese traditional medicine, the volatile constituents of which has been scarcely studied, and their chemical compositions haven't been fully characterized. Previous investigation revealed the characterization of 38 components from $C$. salsa essential oil, 25 compounds from $C$. deserticola oil, with the three major components known as methyl 14-methyl pentadecanoate (13.60\%), ethyl palmitate (12.40\%), and 2,5,6-trimethyloctane (7.61\%). The survey also revealed identification of 21 compounds only from the volatile oil C. tubulosa [1, 20-22]. Furthermore, the biological activity of the volatile constituents hasn't been fully investigated. Hence, our aim is to identify the chemical compositions of the volatile constituents of $C$. tubulosa flowers and estimate their antioxidant and antimicrobial activities.

\section{Materials and Methods}

\subsection{Plant Material}

Cistanche tubulosa (Schenk) Hook. f. (Orobanchaceae) was collected in March 2019 from Sakaka desert, Aljouf, KSA. Identification of the plant was done by Mr. Hamdan Al-Hassan, M.Sc. (Camel and Range Research Center), Aljouf, KSA. A voucher specimen (59-CPJU) was archived in the herbarium of the Pharmacognosy Department, Pharmacy College, Jouf University.

\subsection{Extraction of the Volatile Constituents}

The flowers of $C$. tubulosa were collected in March 2019, carefully washed with running water, and the volatile constituents were extracted by the standard hydro-distillation method with Clevenger apparatus. A $500 \mathrm{~g}$ fresh flowers were cut into small pieces and subjected to hydro-distillation for 5 hours until no more yield was produced. the distillates were separated from the aqueous phase by a $500 \mathrm{~mL}$ volume separating funnel. $\mathrm{NaCl}$ was used to expel the rest of volatile constituents from the aqueous layer by the salting out mechanism. The aqueous phase was shaken several times with $\mathrm{CH}_{2} \mathrm{Cl}_{2}$ to obtain all distillates. The combined extracts were then filtered through a Whatman filter paper (No.40) after being passed over anhydrous $\mathrm{Na}_{2} \mathrm{SO}_{4}$ for dehydration. The product was calculated as $0.36 \%$ total volatiles. The obtained constituents were pale yellow liquid with pleasant odor. It was packed in dry clean and tightly closed opaque bottle and kept in dark at $4^{\circ} \mathrm{C}$ for analysis.

\subsection{Gas chromatography and Gas Chromatography - Mass Spectrometry (GC-MS)}

The model 6890 of an Agilent gas chromatograph supplied with a $120 \mathrm{~m} \times 0.25 \mathrm{~mm}$ i.d. $(\mathrm{df}=$ $0.25 \mu \mathrm{m}$ ) cemented phase HP-5MS stuck silica capillary column (Agilent, Folsom, CA) and flame ionization detector (FID) was applied for volatile extract analysis. The temperature of oven, was adjusted from 60 to $240^{\circ} \mathrm{C}$ at $3^{\circ} \mathrm{C} / \mathrm{min}$. and kept for $50 \mathrm{~min}$. The carrier gas of linear helium feed ratio was 20 $\mathrm{cm} / \mathrm{sec}$. The temperature of injector and detector was $250^{\circ} \mathrm{C}$. 
The volatile constituents were analyzed by Agilent Technologies model 7890B GC interfaced to Agilent 7000D GC/TQ mass detector (GC/MS), and Agilent 7693A autosampler. Ionization at $70 \mathrm{eV}$, HP-5MS column $(120 \mathrm{~m} \times 0.25 \mathrm{~mm}$ i.d). The whole process was conducted at $30 \mathrm{~cm} / \mathrm{s}$ constant velocity of the mobile phase $(\mathrm{He})$ and constant temperature at $250{ }^{\circ} \mathrm{C}$ for both injector and detector. The oven temperature was programmed from 60 to $240^{\circ} \mathrm{C}$ at $3{ }^{\circ} \mathrm{C} / \mathrm{min}$ and retained for $50 \mathrm{~min}$.

Concomitant injection of the sample with a solution of homologous n-hydrocarbons $\left(\mathrm{C}_{8}-\mathrm{C}_{26}\right)$ series under the same conditions were performed determine Kovat's indices values. Identification of the isolated volatiles was done by matching with NIST mass-spectral library data, comparison of Kovat's indices with those of authentic components and with published data. Quantitative determination was carried out according to peak area integration.

\subsection{Antioxidant Activity}

\subsubsection{DPPH Radical Scavenging Assay}

The potential antioxidant activity of the obtained volatile constituents was evaluated by the standard DPPH method, the tert-butylhydroquinone (TBHQ) was applied as a standard antioxidant drug. The measurement of absorption was performed at $\lambda_{\max } 517 \mathrm{~nm}$ on UV-spectrophotometer (HP 8452, UVVIS), all tests were conducted in triplicates and average of the results was calculated [23, 24].

\subsection{2. $\beta$-Carotene Bleaching Assay}

The standard $\beta$-carotene/linoleic acid method was applied for determination of the antioxidant activity of $C$. tubulosa volatile constituents as previously described, relative to the standard antioxidant tert-butylhydroquinone (TBHQ). All tests were measured in triplicates at $\lambda_{\max } 470 \mathrm{~nm}$ over 60 minutes starting from the 0 minute, and the average of the results was calculated [23, 24].

\subsubsection{ABTS Free Radical Assay}

The ABTS [2,2-azino-bis(3-ethylbenzothiazoline-6-sulfonic acid), was applied for the antioxidant determination of $C$. tubulosa volatile constituents as described in the literature [25], in comparison to the standard antioxidant tert-butylhydroquinone (TBHQ). All tests were measured in triplicates at $\lambda_{\max } 734 \mathrm{~nm}$ over 60 minutes starting from the 0 minute, and the average of the results was calculated [26]. The following equation was applied for calculating the free radical scavenging effect in all methods

$\%$ inhibition $=\mathrm{A}($ control $)-\mathrm{A}($ test or standard $) / \mathrm{A}($ control $) \times 100$,

Where, $\mathrm{A}=$ Absorbance

\subsection{Antimicrobial Assay}

\subsubsection{Preparation of Microbial Suspensions}

Nine strains of pathogenic microorganisms which are regarded as main source of several diseases and food intoxication were selected for antimicrobial assay including S. aureus, B. cereus E. fecalis, and L. monocytogenes were employed as G+ve bacteria, E. coli, P. aeruginosa, K. pneumoniae and Salmonella typhimurium as G-ve bacteria. Additionally, C. albicans was employed as fungal strain. The quantitative minimum inhibitory concentration (MIC) method was applied for antimicrobial estimation of the volatile constituents of $C$. tubulosa. The bacterial and fungal suspensions were prepared in the suitable 
broth media for each (Muller Hinton Sabaroud Dextrose for bacteria and fungi, respectively). Incubation of each strain with the proper media was done for $24 \mathrm{~h}$ at $37{ }^{\circ} \mathrm{C}$ for bacteria and $28{ }^{\circ} \mathrm{C}$ for fungi. Following the incubation period and the serial dilutions of the prepared suspensions, certain dilutions were selected according to $0.5 \mathrm{Mc}$-Farlandscale standard for the assay. The standard ciprofloxacin and fluconazole were prepared in $100 \mu \mathrm{g} / \mathrm{mL}$ and applied as antimicrobial and antifungal drugs, respectively [28-30].

\subsubsection{Minimum Inhibitory Concentration Method (MIC)}

A microtiter dilution plate quantitative method was applied, where the minimum inhibitory concentration (MIC) method was applied for assessment of the antimicrobial activity of $C$. tubulosa volatile constituents, against certain microorganisms as mentioned. A sterile 96- micro plate wells was used, where $100 \mu \mathrm{L}$ of the respective microorganisms in concentrations of $(0.5 \mathrm{Mc}$-Farland, about $1 \times 108 \mathrm{cfu} / \mathrm{mL})$ were separately mixed with the obtained distillate in different concentrations (100\%, followed by two fold serial dilutions). Ciprofloxacin and fluconazole were applied as positive antibacterial and antifungal standards respectively, while DMSO was applied as negative control. The microplate with the mixed contents in each well was incubated for $24 \mathrm{~h}$ at $\approx 37^{\circ} \mathrm{C}$ for bacteria and $28{ }^{\circ} \mathrm{C}$ for fungi. The plates were then visualized for any growth precipitation of the tested organisms. All experiments were conducted in triplicates and the MIC was calculated as the lowest concentration that inhibited or hindered the growth of the tested microorganisms [27].

\section{Results and Discussion}

\subsection{Analysis of Volatile Constituents}

In our study, hydrodistillation of C. tubulosa flowers produced $0.36 \%$ pale yellow distillate, with an aromatic fragrant odor and 106 volatile components (table 1) representing $99.29 \%$ of the volatile content. These components were categorized as 5 monoterpenes, 15 sesquiterpenes, 62 light oxygenated compounds representing the largest group compounds and 24 heavy oxygenated compounds. The major components of the volatile constituents were identified as hexanal (15.98\%), trans-sabinyl acetate (12.22\%), allo-aromadendrene (9.30\%), nonanoic acid (6.66\%), 3Z- hexeny-2-methyl butanoate (6.09\%), valeranone $(5.25 \%),(E, E)$ - $\alpha$-Farnesene $(3.18 \%), \alpha$-pinene $(3.06 \%)$, linalool isovalerate $(3.03 \%), \alpha$ humulene (1.8\%), Jasminol (1.58\%), 4-hydroxy benzaldehyde (1.56\%), Geosmin (1.44\%), 3Z- hexenyl isobutanoate $(1.39 \%)$ and geranyl acetone $(1.38 \%)$. But in previous literature studies results, which were published by Jiang and Tu 2009, only 21 volatile components were identified in the essential oil of $C$. tubulosa, 38 components were also characterized from $C$. salsa essential oil, while 25 compounds were identified from the oil of $C$. deserticola, with three major components (methyl 14-methyl pentadecanoate; $13.60 \%$, ethyl palmitate; $12.40 \%$, and 2,5,6-trimethyloctane; 7.61\%) [22, 28]. Identification of the isolated volatiles was done tentatively by matching with NIST mass-spectral library data, furtherly confirmed by comparison of Kovat's indices with those of authentic components as well as with the published data [29-31]. It was found that the calculated KI of identified compounds fall in the KI range of those published in literature. For instance, the calculated and published KI for trans Sabinyl acetate (1287\& 1273-1289), aromadendrene (1444\& 1430-1450), valeranone (1678, 1668-1679), alloaromadendrene (1459\& 1458-1470), $\alpha$-Farnesene (1508\& 1505-1520), hexenyl-2-methyl butanoate (1230\& 1210-1231), $\alpha$-humulene (1455\& 1452-1570), $\alpha$-pinene (971\& 933-982) [32-36]. According to the mentioned KI values, the identified compounds were consistent with those reported in the literature [37-40]. 
Table 1. Volatile constituents of C. tubulosa

\begin{tabular}{|c|c|c|c|c|c|c|}
\hline $\begin{array}{c}\text { Peak } \\
\#\end{array}$ & ${ }^{\mathrm{a} C o n c} \%$ & $\begin{array}{c}\text { Calculated } \\
{ }^{\text {b KI }}\end{array}$ & ${ }^{c}$ KI Data & Compound Name & Type $^{\mathrm{d}}$ & $\begin{array}{l}\text { Identification } \\
\text { Methods }\end{array}$ \\
\hline 1. & 3.06 & 971 & $933-982$ & $\alpha$-pinene & M & KI\&MS $\& S t^{\mathrm{f}}$. \\
\hline 2. & 15.98 & 975 & $932-975$ & Hexanal & LOC & KI\&MS\&St. \\
\hline 3. & 0.01 & 976 & $972-1004$ & Trans pinane & M & KI\&MS \\
\hline 4. & 0.06 & 972 & $952-1019$ & Hexanoic acid & LOC & KI\&MS\&St. \\
\hline 5. & 0.02 & 980 & $974-993$ & Octene-2-ol, 3E & LOC & KI\&MS\&St. \\
\hline 6. & 0.01 & 985 & $982-1002$ & Pentyl furan & LOC & KI\&MS \\
\hline 7. & 0.01 & 987 & $962-993$ & Myrcene & M & KI\&MS\&St. \\
\hline 8. & 0.01 & 994 & $988-1009$ & Butyl butanoate & LOC & KI\&MS \\
\hline 9. & 0.01 & 996 & $987-998$ & Ethyl hexanoate & LOC & KI\&MS\&St. \\
\hline 10. & 0.02 & 1007 & $994-1025$ & Hexyl acetate & LOC & KI\&MS\&St. \\
\hline 11. & 0.05 & 1014 & $1013-1020$ & Heptadienol (2E, 4E) & LOC & KI\&MS \\
\hline 12. & 0.05 & 1025 & $1020-1043$ & $\begin{array}{l}\text { Cyclo-pentanedione (3- } \\
\text { methyl-1,2) }\end{array}$ & LOC & KI\&MS\&St. \\
\hline 13. & 0.05 & 1051 & $1042-1056$ & $\gamma$-hexalactone & LOC & KI\&MS \\
\hline 14. & 0.01 & 1058 & $1049-1060$ & Pentyl isobutanoate & LOC & KI\&MS \\
\hline 15. & 0.28 & 1065 & $1050-1070$ & Thiophene (2-butyl) & LOC & KI\&MS \\
\hline 16. & 0.05 & 1083 & $1060-1083$ & Camphenilone & LOC & KI\&MS \\
\hline 17. & 0.03 & 1084 & 1079-1089 & Allyl hexanoate & LOC & KI\&MS \\
\hline 18. & 0.24 & 1092 & $1088-1095$ & Isobutyl tiglate & LOC & KI\&MS \\
\hline 19. & 0.04 & 1093 & $1080-1101$ & Linalool & LOC & KI\&MS \\
\hline 20. & 0.18 & 1096 & $1096-1110$ & Dimethyl styrene $(2,5)$ & M & KI\&MS \\
\hline 21. & $0.06 ; 2$ & 1105 & $1103-1140$ & Phenyl propanol (2) & LOC & KI\&MS \\
\hline 22. & 0.08 & 1129 & $1124-1135$ & Chrysanthenone & LOC & KI\&MS \\
\hline 23. & 0.19 & 1138 & $1128-1140$ & Ocimene (allo) & LOC & KI\&MS \\
\hline 24. & 1.39 & 1141 & 1130-1142 & $\begin{array}{l}\text { Hexenyl isobutanoate } \\
\text { (3Z) }\end{array}$ & LOC & KI\&MS \\
\hline 25 . & 0.05 & 1149 & $1145-1455$ & Myrcenone & LOC & KI\&MS \\
\hline 26. & 0.02 & 1151 & $1149-1165$ & Thujanol (neoiso-3-) & LOC & KI\&MS \\
\hline 27. & 0.02 & 1161 & $1153-1167$ & Nerol oxide & LOC & KI\&MS \\
\hline 28. & 0.04 & 1163 & $1159-1170$ & $\begin{array}{l}\text { Hexadienol propanoate } \\
\qquad(2 \mathrm{E}, 4 \mathrm{E})\end{array}$ & LOC & KI\&MS \\
\hline 29. & 6.09 & 1230 & $1210-1231$ & $\begin{array}{l}\text { Hexenyl 2-methyl } \\
\text { butanoate (3Z) }\end{array}$ & LOC & KI\&MS\&St. \\
\hline 30. & 0.62 & 1236 & $1234-1250$ & $\begin{array}{l}\text { Linalool acetate } \\
\text { (tetrahydro) }\end{array}$ & LOC & KI\&MS \\
\hline 31. & 0.98 & 1239 & $1234-1250$ & Citronet & LOC & KI\&MS \\
\hline 32. & 0.01 & 1242 & $1236-1244$ & Carvotanacetone & LOC & KI\&MS \\
\hline 33. & 0.02 & 1259 & $1240-1265$ & $\begin{array}{c}\text { Sabinene hydrate acetate } \\
\text { (trans) }\end{array}$ & LOC & KI\&MS \\
\hline 34. & 0.04 & 1269 & $1264-1285$ & Phenol (2-(1E)-propenyl-) & LOC & KI\&MS \\
\hline 35. & 6.66 & 1271 & $1267-1273$ & Nonanoic acid & LOC & KI\&MS\&St. \\
\hline
\end{tabular}




\begin{tabular}{|c|c|c|c|c|c|c|}
\hline $\begin{array}{c}\text { Peak } \\
\#\end{array}$ & ${ }^{\mathrm{a}}$ Conc.\% & $\begin{array}{c}\text { Calculated } \\
\text { bKI }\end{array}$ & 'KI Data & Compound Name & Type $^{d}$ & $\begin{array}{l}\text { Identification } \\
\text { Methods }\end{array}$ \\
\hline 36. & 0.79 & 1273 & $1268-1298$ & Decen-1-ol (2E)- & LOC & KI\&MS \\
\hline 37. & 12.22 & 1287 & $1273-1289$ & Sabinyl acetate (trans) & LOC & KI\&MS\&St. \\
\hline 38. & 1.05 & 1292 & $1270-1293$ & Verbinyl acetate (trans) & LOC & KI\&MS\&St. \\
\hline 39. & 0.06 & 1299 & $1298-1320$ & Pinocarvyl acetate & LOC & KI\&MS \\
\hline 40. & 0.14 & 1300 & $1299-1310$ & Terpinen-4-ol acetate & LOC & KI\&MS \\
\hline 41. & 0.86 & 1302 & $1270-1315$ & Necrodol acetate (cis- $\square$ ) & LOC & KI\&MS \\
\hline 42. & 0.05 & 1319 & $1318-1330$ & Pinanediol (cis-2,3) & LOC & KI\&MS \\
\hline 43. & 0.34 & 1321 & $1282-1325$ & Verbenol acetate (neo) & LOC & KI\&MS \\
\hline 44. & 0.95 & 1326 & $1305-1332$ & Myrtenyl acetate & LOC & KI\&MS \\
\hline 45. & 1.58 & 1331 & $1315-1340$ & Jasminol & LOC & KI\&MS\&St. \\
\hline 46. & 0.15 & 1334 & $1330-1350$ & $\begin{array}{l}\text { Menth-1-ene-7-al (3-oxo- } \\
\text { p) }\end{array}$ & LOC & KI\&MS \\
\hline 47. & 0.06 & 1337 & $1333-1441$ & Hasmigone (E) & LOC & KI\&MS \\
\hline 48. & 0.16 & 1339 & $1334-1442$ & Linalool propanoate & $\mathrm{HOC}$ & KI\&MS \\
\hline 49. & 0.86 & 1341 & $1335-1345$ & Elemene $(\delta)-$ & $\mathrm{S}$ & KI\&MS \\
\hline 50. & 0.95 & 1342 & $1339-1350$ & Carvyl acetate (trans) & LOC & KI\&MS \\
\hline 51. & 0.01 & 1344 & $1342-1370$ & Verbenol acetate & LOC & KI\&MS \\
\hline 52. & 0.02 & 1347 & $1343-1367$ & Piperitol acetate (trans) & LOC & KI\&MS \\
\hline 53. & 1.56 & 1353 & $1353-1355$ & Hydroxybenzaldehyde (4) & LOC & KI\&MS\&St. \\
\hline 54. & 0.06 & 1360 & $1301-1370$ & Undecanol (2E) & LOC & KI\&MS \\
\hline 55. & $0.03 ; 2$ & 1366 & $1366-1371$ & Piperitenone oxide & LOC & KI\&MS \\
\hline 56. & 0.05 & 1368 & $1367-1373$ & Furfuryl hexanoate & LOC & KI\&MS \\
\hline 57. & 0.25 & 1375 & $1373-1385$ & Linalool isobutanoate & $\mathrm{HOC}$ & KI\&MS \\
\hline 58. & 0.06 & 1380 & $1377-1380$ & Ethyl-(4E)-decenoate & LOC & KI\&MS \\
\hline 59. & 0.14 & 1387 & $1382-1387$ & Hexyl hexanoate & $\mathrm{HOC}$ & KI\&MS \\
\hline 60. & 0.01 & 1390 & $1385-1398$ & Myrtanol acetate (trans) & LOC & KI\&MS \\
\hline 61. & 0.10 & 1392 & $1389-1398$ & Damascene (Z) - $\beta$ & LOC & KI\&MS \\
\hline 62. & 0.08 & 1395 & $1399-1407$ & $\begin{array}{l}\text { Neptalactone }(4 \mathrm{a} \alpha, 7 \alpha, \\
7 \mathrm{a} \beta)\end{array}$ & LOC & KI\&MS \\
\hline 63. & 0.5 & 1398 & $1393-1405$ & Vanillin & LOC & KI\&MS\&St. \\
\hline 64. & 1.44 & 1399 & $1399-1402$ & Geosmin & LOC & KI\&MS \\
\hline 65. & 0.45 & 1404 & $1400-1410$ & $n$-Tetradecane & M & KI\&MS\&St. \\
\hline 66. & 0.74 & 1409 & $1407-1440$ & Decyl acetate & $\mathrm{HOC}$ & KI\&MS \\
\hline 67. & 0.06 & 1411 & $1409-1420$ & Gurjunene $(\alpha)$ & $S$ & KI\&MS. \\
\hline 68. & 0.08 & 1420 & $1417-1460$ & Ethyl-(2E)-decenoate & LOC & KI\&MS\&St. \\
\hline 69. & 0.56 & 1433 & $1430-1450$ & $\begin{array}{l}\text { Isoamyl 3-(2-furan) } \\
\text { propionate }\end{array}$ & $\mathrm{HOC}$ & KI\&MS \\
\hline 70. & 0.05 & 1434 & $1417-1445$ & Caryophyllene (E) & $S$ & KI\&MS\&St. \\
\hline 71. & 0.53 & 1438 & $1420-1450$ & Cascarilladiene & $S$ & KI\&MS \\
\hline 72. & 0.92 & 1440 & $1438-1460$ & Methyl butyl benzoate & LOC & KI\&MS\&St. \\
\hline 73. & 2.80 & 1444 & $1430-1450$ & Aromadendrene & S & KI\&MS\&St. \\
\hline 74. & 1.8 & 1455 & $1452-1570$ & Humulene $(\alpha)$ & $\mathrm{S}$ & KI\&MS\&St. \\
\hline 75. & 1.38 & 1456 & $1453-1570$ & Geranyl acetone & LOC & KI\&MS\&St. \\
\hline
\end{tabular}




\begin{tabular}{|c|c|c|c|c|c|c|}
\hline $\begin{array}{c}\text { Peak } \\
\#\end{array}$ & ${ }^{\mathrm{a}}$ Conc.\% & $\begin{array}{c}\text { Calculated } \\
{ }^{\mathbf{b}} \mathrm{KI}\end{array}$ & 'KI Data & Compound Name & Type $^{\text {d }}$ & $\begin{array}{c}\text { Identification } \\
\text { Methods }\end{array}$ \\
\hline 76. & 9.30 & 1459 & $1458-1470$ & Aromadendrene (allo) & $S$ & KI\&MS\&St. \\
\hline 77. & 3.03 & 1470 & $1466-1495$ & Linalool isovalerate & HOC & KI\&MS \\
\hline 78. & 0.26 & 1473 & $1465-1483$ & Jasmone lactone & LOC & KI\&MS \\
\hline 79. & 0.27 & 1474 & $1472-1515$ & Terpenyl isobutanoate $(\alpha)$ & HOC & KI\&MS \\
\hline 80. & 0.01 & 1479 & 1474-1499 & Acoradiene (-10-epi- $\beta-)$ & S & KI\&MS. \\
\hline 81. & 0.25 & 1483 & $1481-1499$ & Curcumene $(\gamma)$ & S & KI\&MS\&St. \\
\hline 82. & 0.04 & 1485 & $1481-1520$ & Menthyl lactate & $\mathrm{HOC}$ & KI\&MS \\
\hline 83. & 0.14 & 1490 & $1487-1516$ & Ionone $(\mathrm{E})-\beta$ - & LOC & KI\&MS \\
\hline 84. & 0.42 & 1491 & $1486-1515$ & Menthyl lactate (iso) & $\mathrm{HOC}$ & KI\&MS \\
\hline 85. & 0.05 & 1496 & $1494-1523$ & Maltyl isobutyrate & LOC & KI\&MS \\
\hline 86. & 0.01 & 1498 & $1496-1524$ & Valencene & S & KI\&MS \\
\hline 87. & 0.39 & 1504 & $1501-1525$ & Aciphyllene & S & KI\&MS \\
\hline 88. & 3.18 & 1508 & $1505-1520$ & Farnesene $(E, E)-\alpha-$ & $S$ & KI\&MS\&St. \\
\hline 89. & 1.07 & 1514 & $1513-1518$ & Cadinene $(\gamma)$ & $S$ & KI\&MS\&St. \\
\hline 90. & 0.15 & 1518 & $1514-1520$ & Italicene epoxide (iso) & $\mathrm{HOC}$ & KI\&MS \\
\hline 91. & 0.18 & 1520 & $1518-1526$ & $\begin{array}{l}\begin{array}{l}\text { Silphiperfolan-6- } \beta \text {-ol (7- } \\
\text { epi-) }\end{array}\end{array}$ & HOC & KI\&MS \\
\hline 92. & 0.06 & 1522 & $1519-1528$ & Dihydroagarofuran (cis) & HOC & KI\&MS \\
\hline 93. & 0.59 & 1524 & $1520-1529$ & Ionone (6-methyl- $\alpha$-) & $\mathrm{HOC}$ & KI\&MS \\
\hline 94. & 0.34 & 1532 & $1529-1535$ & Kessane & HOC & KI\&MS \\
\hline 95. & 0.30 & 1537 & $1533-1540$ & Cadina-1,4-diene (trans) & S & KI\&MS \\
\hline 96. & 0.20 & 1539 & $1533-1545$ & Cubebol (10-epi) & HOC & KI\&MS \\
\hline 97. & 0.02 & 1540 & $1537-1546$ & Cadinene $(\alpha)$ & $S$ & KI\&MS \\
\hline 98. & 0.03 & 1545 & $1539-1549$ & Copaen-11-ol $(\alpha)$ & HOC & KI\&MS \\
\hline 99. & 0.56 & 1549 & $1547-1555$ & Elemol & HOC & KI\&MS\&St. \\
\hline 100. & 0.02 & 1638 & $1632-1638$ & $\begin{array}{l}\text { Isoborneol (8- } \\
\text { isobutyryloxy) }\end{array}$ & $\mathrm{HOC}$ & KI\&MS \\
\hline 101. & 0.53 & 1647 & $1639-1455$ & $\begin{array}{l}\text { Aromadendrene epoxide } \\
\text { (allo) }\end{array}$ & $\mathrm{HOC}$ & KI\&MS \\
\hline 102. & 0.08 & 1648 & $1639-1650$ & Cedrenal (1,7-diepi- $\alpha)$ & $\mathrm{HOC}$ & KI\&MS \\
\hline 103. & 0.12 & 1649 & $1467-1654$ & Methyl jasmonate (Z) & HOC & KI\&MS \\
\hline 104. & 0.86 & 1662 & $1660-1675$ & Intermedeol (neo) & $\mathrm{HOC}$ & KI\&MS \\
\hline 105. & 1.24 & 1675 & $1668-1683$ & Bisabolol (epi- $\beta-$ ) & HOC & KI\&MS\&St. \\
\hline \multirow[t]{2}{*}{106.} & 5.25 & 1678 & $1668-1679$ & Valeranone & HOC & KI\&MS\&St. \\
\hline & Total & & & 99.29 & & \\
\hline
\end{tabular}

${ }^{\mathrm{a}}$ Conc.\%: the percent of concentrations based on peak area integration.

bKI Confirmed by comparison with Kovat's index on DB5 column (Adams, 2017).

${ }^{\mathrm{c}} \mathrm{KI}$ data -Kovats index data reported in plants essential oils on DB5 (Ref. 31-40) and non-polar column (www.webbook.nist.gov)

${ }^{\mathrm{d}} \mathrm{M}$, monoterpene; LOC, light oxygenated compounds; HOC, heavy oxygenated compounds.

${ }^{\mathrm{e}}$ Tentative identification by comparison with data obtained from NIST mass spectra library.

${ }^{\mathrm{f}}$ Confirmed by comparison with mass spectrum of authentic compound. 


\subsection{Results of Antioxidant Assay}

DPPH, ABTS and $\beta$-carotene assays were applied to investigate the antioxidant activity of $C$. tubulosa volatile constituents, the results exhibited reliable antioxidant activity of the tested volatiles (Table 2). The current study indicated that the scavenging ability of $C$. tubulosa volatile constituents at various concentrations $(\mu \mathrm{g} / \mathrm{mL})$ ranged from $26.08 \%$ to $62.40 \%$ for the DPPH assay, while from 25.71 to $63.29 \%$ and 27.31 to $62.72 \%$ at $(20-80) \mu \mathrm{g} / \mathrm{mL}$ for the ABTS and $\beta$-carotene testing systems, respectively, compared to the standard TBHQ antioxidant drug, that showed 43.15 to $78.62 \%, 41.32$ to $77.56 \%$ and 42.21 to $79.23 \%$ at $(20-80) \mu \mathrm{g} / \mathrm{mL}$ for the DPPH, ABTS and $\beta$-carotene testing systems, respectively. The promising antioxidant activity may be attributed to the presence of highly active complex mixture in the distillate as allo-aromadendrene (9.3\%), valeranone (5.25\%), (E, E)- $\alpha$-Farnesene (3.18\%) and $\alpha$-pinene $(3.06 \%)$ which were supposed to have pronounced impact on the antioxidant activity. According the literature survey, the target biological activity of the volatile constituents may be attributed to the existing mixture of terpenoid and phenolic components, which known to have antimicrobial and antioxidant activities and can enhance or synergizes the target activities [32].

Table 2. Antioxidant activity of hydro-distilled (HD) C. tubulosa volatile constituents determined by DPPH, ABTS and by $\beta$ carotene/ Linoleic acid assays compared to the synthetic antioxidant TBHQ

\begin{tabular}{ccccccc}
\hline $\begin{array}{c}\text { Sample } \\
\text { concentration }\end{array}$ & \multicolumn{2}{c}{ \% Inhibition by DPPH } & \multicolumn{2}{c}{ \% Inhibition by ABTS } & \multicolumn{2}{c}{$\begin{array}{c}\text { \% Inhibition by } \boldsymbol{\beta} \\
\text { carotene/ Linoleic acid }\end{array}$} \\
\hline & EO & TBHQ & EO & TBHQ & EO & TBHQ \\
$20(\mu \mathrm{g} / \mathrm{mL})$ & $26.08 \pm 1.8$ & $43.15 \pm 2.1$ & $25.71 \pm 1.9$ & $41.32 \pm 2.0$ & $27.31 \pm 1.9$ & $42.21 \pm 2.1$ \\
$40(\mu \mathrm{g} / \mathrm{mL})$ & $39.56 \pm 2.0$ & $64.41 \pm 1.8$ & $38.43 \pm 2.0$ & $63.63 \pm 1.9$ & $38.02 \pm 2.0$ & $65.46 \pm 2.2$ \\
$60(\mu \mathrm{g} / \mathrm{mL})$ & $51.22 \pm 2.1$ & $71.47 \pm 2.0$ & $52.14 \pm 1.8$ & $70.73 \pm 2.1$ & $51.86 \pm 2.2$ & $72.34 \pm 1.9$ \\
$80(\mu \mathrm{g} / \mathrm{mL})$ & $62.40 \pm 2.0$ & $78.62 \pm 2.2$ & $63.29 \pm 2.1$ & $77.56 \pm 2.3$ & $62.72 \pm 2.1$ & $79.23 \pm 2.1$ \\
\hline
\end{tabular}

${ }^{a}$ Values characterize averages \pm standard deviations for triplicate experiments. Means with the same letter within the same row are not significantly different $(P>0.05)$.

\subsection{Results of Antimicrobial Assay}

The MIC (minimum inhibitory concentration) method was applied to test the antimicrobial activity of the volatile constituents of $C$. tubulosa against nine pathogenic microorganisms of animal origin was investigated. The distillate exhibited strong activity against $S$. aureus with MIC $2.23 \mathrm{mg} / 100 \mathrm{~mL}$ and moderate effect against $C$. albicans $(\mathrm{MIC}=4.36 \mathrm{mg} / 100 \mathrm{~mL}$ ), table 3 .

Table 3. Antimicrobial activity of hydro-distilled constituents of C. tubulosa

\begin{tabular}{ccccc}
\hline \multirow{2}{*}{ Type of strain } & Strain & Minimum inhibitory concentration (MIC) mg/100 mL \\
& EO & Ciprofloxacin & Fluconazole \\
\hline \multirow{4}{*}{ Gram-positive } & Staphylococcus aureus & $2.23 \pm 0.52$ & $0.185 \pm 0.09$ & NT \\
& Enterococcus faecalis & $12.47 \pm 0.48$ & $0.096 \pm 0.02$ & NT \\
& Bacillus cereus & $15.68 \pm 0.43$ & $0.182 \pm 0.011$ & NT \\
& Listeria monocytogenes & $6.06 \pm 0.54$ & $0.093 \pm 0.07$ & NT \\
& Escherichia coli & $18.35 \pm 0.39$ & $0.184 \pm 0.05$ & NT \\
Gram-negative & Salmonella typhimurium & $26.71 \pm 0.41$ & $0.095 \pm 0.07$ & NT \\
& Pseudomonas aeruginosa & $23.53 \pm 0.32$ & $0.096 \pm 0.03$ & NT \\
Fungi & Klebsiella pneumonia & $31.61 \pm 0.49$ & $0.188 \pm 0.06$ & NT \\
& Candida albicans & $4.36 \pm 0.38$ & NT & $0.191 \pm 0.01$ \\
\hline
\end{tabular}

NT: not tested 


\section{Conclusion}

The volatile components of $C$. tubulosa or the commonly known as Desert Ginseng are composed mainly of hexanal $(15.98 \%)$, trans-sabinyl acetate $(12.22 \%)$, allo-aromadendrene $(9.30 \%)$, nonanoic acid (6.66\%), 3Z-hexeny-2-methyl butanoate $(6.09 \%)$, valeranone $(5.25 \%),(E, E)$ - $\alpha$-Farnesene $(3.18 \%), \alpha$ pinene (3.06\%), linalool isovalerate (3.03\%) and $\alpha$-humulene $(1.8 \%)$, which were characterized by their retention times and the fragmentation pattern for each, in the GC-MS chromatogram, as well as comparison to the literature. These components showed promising antioxidant effect at concentration of $80 \mu \mathrm{g} / \mathrm{mL}$ and comparatively similar results upon using three methods of assay. It also exhibited strong antimicrobial and antifungal activities against $S$. aureus $L$. monocytogenes and $C$. albicans compared to ciprofloxacin and fluconazole.

\section{Acknowledgments}

The authors extend their appreciation to the Deanship of Scientific Research at Jouf University for funding this work through research grant No (DSR2020-04-453), and to Taif University Researchers Supporting Project number (TURSP-2020/56), Taif University, Taif, Saudi Arabia.

\section{Conflict of Interest}

The authors declare no conflicts of interest.

\section{ORCID}

Arafa Musa: 0000-0003-0979-556X

Khaled F. El-Massry: 0000-0001-9029-2243

Ahmed H. El-Ghorab: 0000-0002-5500-3124

Amr Farouk: 0000-0002-5261-2397

Hazim M. Ali: 0000-0003-0781-0850

Mohamed A Abdelgawad: 0000-0001-9035-5638

Ibrahim A. Naguib: 0000-0002-5923-1466

Ehab M. Mostafa: $\underline{0000-0001-8841-9786}$

\section{References}

[1] Y. Jiang and P.-F. Tu (2009). Analysis of chemical constituents in Cistanche species, J. Chrom. A. 1216(11), 1970-1979.

[2] L. Zhiming, L. Huinuan, G. Long, G. Jingwen and T. Chi-Meng (2016). Herba Cistanche (Rou CongRong): One of the best pharmaceutical gifts of traditional Chinese medicine, Front. Pharmacol. 7, 41.

[3] C. Gu, X. Yang and L. Huang (2016). Cistanches Herba: a neuropharmacology review, Front. Pharmacol. 7, 289.

[4] E. M. Abdallah (2017). Antimicrobial evaluation of flowering stalks of Cistanche violacea, a holoparasitic plant collected from arid region in Qassim, Saudi Arabia, Pharma. Biol. Eval. 4(6), 239-244.

[5] K. Zwe-Ling, J. Athira, K. Fan-Chi, H. Jia-Ling and C. Shu-Chun (2018). Effect of Cistanche tubulosa extracts on male reproductive function in streptozotocin-nicotinamide-induced diabetic rats, Nutrients 10(10), 1562.

[6] W. Lin-lin, D. Hui, Y. He-shui, L. Qing-hai, Z. Li-juan and S. Xin-bo (2015). Cistanches Herba: chemical constituents and pharmacological effects, Chin. Herb. Med. 7(2), 135-142.

[7] F. Changshuang, L. Jinyu, A. Adila, X. Lijie, Y. Yi, C. Qiuyan, L. Jie, W. Xinhui and L. Jinyao (2019). Cistanche tubulosa phenylethanoid glycosides induce apoptosis in Eca-109 cells via the mitochondria-dependent pathway, Oncol. Lett. 17(1), 303-313.

[8] Q. Xu, W. Fan, S.-F. Ye, Y.-B. Cong, W. Qin, S.-Y. Chen and J. Cai (2016). Cistanche tubulosa protects dopaminergic neurons through regulation of apoptosis and glial cell-derived neurotrophic factor: in vivo and in vitro, Front. Aging Neurosci. 8, 295. 
[9] N. Wang, S. Ji, H. Zhang, S. Mei, L. Qiao and X. Jin (2017). Herba Cistanches: anti-aging, Aging Diseas. $\mathbf{8}(\mathbf{6}), 740$.

[10] A. Bougandoura, B. D'Abrosca, S. Ameddah, M. Scognamiglio, R. Mekkiou, A. Fiorentino, S. Benayache and F. Benayache (2016). Chemical constituents and in vitro anti-inflammatory activity of Cistanche violacea Desf.(Orobanchaceae) extract, Fitoterapia 109, 248-253.

[11] Z.Jiang, J. Wang, X. Li and X. Zhang (2016). Echinacoside and Cistanche tubulosa (Schenk) R. wight ameliorate bisphenol A-induced testicular and sperm damage in rats through gonad axis regulated steroidogenic enzymes, J. Ethnopharmacol. 193, 321-328.

[12] C.J. Wu, M.Y. Chien, N.H. Lin, Y.C. Lin, W.Y. Chen, C.H. Chen and J.T.C. Tzen (2019). Echinacoside isolated from Cistanche tubulosa putatively stimulates growth hormone secretion via activation of the ghrelin receptor, Molecules 24(4),720.

[13] X. Wang and Y. Guo (2017). Rapidly simultaneous determination of six effective components in Cistanche tubulosa by near infrared spectroscopy, Molecules 22(5), 843.

[14] A.E. Al-Snafi (2020). Bioactive metabolites and pharmacology of Cistanche tubulosa-A review. IOSR J. Pharm. 10(1), 37-46.

[15] D. Wang, H. Wang and L. Gu (2017). The antidepressant and cognitive improvement activities of the traditional Chinese herb Cistanche, Evid. Based Complem. Altern. Med. 2017, 3925903.

[16] A. E. Al-Snafi (2016). Immunological effects of medicinal plants: A review (part 2), Immun. Endocr. Metab. Agents Med. Chem. 16(2), 100-121.

[17] A. S. Al-Menhali, S. A. Jameela, A. A. Latiff, M. A. Elrayess, M. Alsayrafi and M. Jaganjac (2017). Cistanche tubulosa induces reactive oxygen species-mediated apoptosis of primary and metastatic human colon cancer cells, J. Applied Pharm. Sci. 7(5), 039-045.

[18] H. Shimoda, J. Tanaka, Y. Takahara, K. Takemoto, S.J. Shan and M.H. Su (2009). The hypocholesterolemic effects of Cistanche tubulosa extract, a Chinese traditional crude medicine, in mice, Amer. J. Chin. Med. 37(6), 1125-1138.

[19] X.Y. Wang, R. Xu, J. Chen, J.Y. Song, S.G. Newmaster, J.P. Han, Z. Zhang and S.L. Chen (2018). Detection of Cistanches herba (Rou Cong Rong) medicinal products using species-specific nucleotide signatures, Front. Plant Sci. 9, 1643.

[20] N.S. Du, S.H. Qu, X.D. Re, J.T. Ni, H.Q. Zhang and H.J. Yan (1988). Study on the composition of the essential oil from Cistanche salsa G, Youji Huax. 8, 522-525.

[21] C. Formisano, D. Rigano, F. Senatore, M. SJ Simmonds, A. Bisio, M. Bruno and S.Rosselli (2008). Essential oil composition and antifeedant properties of Bellardia trixago (L.) All.(sin. Bartsia trixago L.)(Scrophulariaceae), Biochem. System. Ecol. 36(5-6), 454-457.

[22] S.J. Roudbaraki and D. Nori-Shargh (2016). The volatile constituent analysis of Orobanche alba Stephan from Iran, Current Anal. Chem. 12(5), 496-499.

[23] A.Baran, E. Karakılıç, Ö. Faiz and F. Özen (2020). Synthesis of chalcone-containing zinc and cobalt metallophthalocyanines; investigation of their photochemical, DPPH radical scavenging and metal chelating characters, Org. Commun.. 13(2), 65-78.

[24] A. Musa, N.S. Al-muaikel and M.S. Abdel-Bakky (2016). Phytochemical and pharmacological evaluations of ethanolic extract of Bassia eriophora, Der Pharm Chem. 8(12), 169-178.

[25] M. M. Ramadan, M. M. Ali, K. Z. Ghanem and A. H. El-Ghorab (2015). Essential oils from Egyptian aromatic plants as antioxidant and novel anticancer agents in human cancer cell lines, Gras. y Aceites 66(2), $\mathrm{e} 080$.

[26] Z.Q. He, X.Y. Shen, Z.Y. Cheng, R.L. Wang, P.X. Lai and X. Xing (2020). Chemical composition, antibacterial, antioxidant and cytotoxic activities of the essential oil of Dianella ensifolia, Rec. Nat. Prod. 14(2), 160-165.

[27] A. Hamed, B. Mahmoud, M. Samy, E. M. Mostafa, A. Wanas, M. Radwan, M. Elsohly and M. Kamel (2019). Phytochemical and antimicrobial studies of Markhamia platycalyx (Baker) Sprague leaves, Trop. J. Pharm. Res. 18, 2623-263.

[28] M. M. Ghoneim, A. Musa, A. A. El-Hela and K. M. Elokely (2018). Evaluation and understanding the molecular basis of the antimethicillin-resistant Staphylococcus aureus activity of secondary metabolites isolated from Lamium amplexicaule, Pharmacog. Mag. 14(55), S3-S7.

[29] M.A Abdelgawad, A. M. Mohamed, A. Musa, E. M. Mostafa and H. M. Awad (2018). Synthesis, chromatographic separation and antimicrobial evolution of new azoquinoline-8-ol, J. Pharm. Sci. Res. 10(6), 1314-1318.

[30] A. Musa (2019). Chemical constituents, antimicrobial and antiinflammatory evaluations of various extracts ofsuaeda vera forssk. growing in Saudi Arabia, Int. J. Pharm. Res. 11(4), 962-967.

[31] R. P. Adams (2017). Identification of essential oil components by gas chromatography/mass spectrometry, ed. 4.1., Allured publishing corporation Carol Stream. 
[32] B. F. M. T. Andrade, L. N. Barbosa, I. S. Probst and A. F. Júnior (2014). Antimicrobial activity of essential oils, J. Essent. Oil Res. 26(1), 34-40.

[33] V. Babushok, P. Linstrom and I. Zenkevich (2011). Retention indices for frequently reported compounds of plant essential oils, J. Phys. and Chem. Ref. Data 40(4), 043101.

[34] J. Alencar, A. Craveiro and F.d.A. Matos (1984). Kovats' indices as a preselection routine in mass spectra library searches of volatiles, J. Nat. Prod. 47(5), 890-892.

[35] M.Y. Tian, X.G. Zhao, X.H. Wu, Y. Hong, Q. Chen, X.L. Liu and Y. Zhou (2020). Chemical composition, antibacterial and cytotoxic activities of the essential oil from Ficus tikoua Bur, Rec.Nat.Prod. 14, 219-224.

[36] A. Judzentiene, F. Tomi and J. Casanova (2009). Analysis of essential oils of Artemisia absinthium L. from Lithuania by CC, GC (RI), GC-MS and ${ }^{13}$ C NMR, Nat. Prod. Comm. 4(8), 1934578 X0900400820.

[37] P. H. Ribeiro, L. M. Santos, C. AG. Camara, F. S. Born and C. W. Fagg (2016). Seasonal chemical compositions of the essential oils of two eugenia species and their acaricidal properties, Quim. Nova. 39, 38-43.

[38] J. Calva, J.M. Castillo, N. Bec, J. Ramirez, J.M. Andrade, C. Larroque and C. Armijos (2019). Chemical composition, enantiomeric distribution and AChE-BChE activities of the essential oil of Myrteola phylicoides (Benth) Landrum from Ecuador, Rec. Nat. Prod. 13, 355-362.

[39] E. M. Mostafa (2020). Exploration of aurora B and cyclin-dependent kinase 4 inhibitors isolated from scorzonera tortuosissima Boiss. and their docking studies, Pharmacog. Mag. 16 (96), 258-263.

[40] J. Yan, X.B. Liu, W.-W. Zhu, X. Zhong, Q. Sun and Y.-Z. Liang (2015). Retention indices for identification of aroma compounds by GC: Development and application of a retention index database, Chromatographia 78(1-2), 89-108.

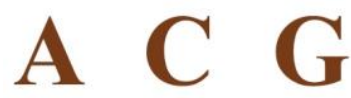

publications

(C) 2021 ACG Publications 